\title{
Improvement of Modal Matching Image Objects in Dynamic Pedobarography using Optimization Techniques
}

\author{
João Manuel R. S. Tavares ${ }^{*}$ and Luísa Ferreira Bastos ${ }^{+}$ \\ * Laboratório de Óptica e Mecânica Experimental, Instituto de Engenharia Mecânica e Gestão Industrial / \\ Departamento de Engenharia Mecânica e Gestão Industrial, Faculdade de Engenharia da Universidade do Porto, \\ Rua Dr. Roberto Frias, s/n, 4200-465, Porto, PORTUGAL \\ + Laboratório de Óptica e Mecânica Experimental, Instituto de Engenharia Mecânica e Gestão Industrial, \\ Rua Dr. Roberto Frias, s/n, 4200-465, Porto, PORTUGAL
}

Received 20 December 2004; accepted 9 March 2005

\begin{abstract}
This paper presents an improved approach for matching objects represented in dynamic pedobarography image sequences, based on finite element modeling, modal analysis and optimization techniques.

In this work, the determination of correspondences between objects data points is improved by using optimization techniques and, because the number of data points of each object is not necessary the same, a new algorithm to match the excess points is also proposed. This new matching algorithm uses a neighbourhood criterion and can overcome some disadvantages of the usual "one to one" matching.

The considered approach allows the determination of correspondences between 2D or 3D objects data points, and is here apply in dynamic pedobarography images.
\end{abstract}

Key Words: Computacional Vision, Deformable Objects, Dynamic Pedobarography, FEM, Matching, Medical Imaging, Modal Analysis, Optimization Techniques.

\section{Introduction}

In several areas of Computational Vision, one of the main problems consists in the determination of correspondences between objects represented in different images, and on the computation of robust canonical descriptors that can be used for their recognition.

In this paper, is presented a methodology to address the above problem, based on an approach initially proposed by Sclaroff [1], [2], and in this work improved by using optimization algorithms in the matching phase. A new algorithm to determine the correspondences between excess models nodes is also proposed, and can be used when the objects to be matched are represented by different number of data points. With this new algorithm, we can successfully overcome some disadvantages of the usual "one to one" matching as, for example, loss of information along image sequences.

The application of the proposed matching methodology on deformable objects represented in dynamic pedobarography image sequences leads to very promising results, and will be discussed in this paper.

Correspondence to: <tavares@fe.up.pt>

Recommended for acceptance by Perales F., Draper B.

ELCVIA ISSN: 1577-5097

Published by Computer Vision Center / Universitat Autonoma de Barcelona, Barcelona, Spain 
The following sections present a brief introduction to the background problem, the dynamic pedobarography principle, the used object models, the proposed matching methodology, experimental results obtained on deformable objects represented in dynamic pedobarography images and some conclusions.

\subsection{Background}

There is an eigen methods class [2] that derives its parameterization directly from objects data shape. Some of these techniques also try to determine, explicitly and automatically, the correspondences between characteristic points' sets, while others try to match images using more global approaches instead of local ones. Each eigen method decomposes the object deformation in an orthogonal and ordered base.

Usually, solution methods for the matching problem include several restrictions that prevent inadequate matches according to some criteria, as for example: order [3], [4]; rigidity [3], [4]; unicity [5]; visibility [6]; and proximity [2]. Some of these methods are image correlation (it is presumed that the images are similar) [7], point proximity [8], and smoothness of disparity fields [3].

The matching problem can also be interpreted as an optimization problem, in which the objective function can, for example, depend on any criteria mentioned in the previous paragraph, and the restrictions considered must form a non-empty space of possible solutions. To solve this optimization problem, it can be used dynamic programming [3], graphs [4] and convex minimization [7]. Non-optimal approaches include, for example, greedy algorithms [9], simulated annealing [10], relaxation [5], etc.

To determine correspondences between two objects, Belongie [11] considered shapes context and a similar optimization technique to the one used in this work. Although shape description algorithms have usually a higher computational efficiency, the modeling methodology considered in this work as the major advantage of attributing a physical behaviour to each object to be matched, through the consideration of a virtual elastic material.

\section{Dynamic Pedobarography}

Pedobarography refers to measuring and visualizing the distribution of pressure under the foot sole. The recording of pedobarographic data along the duration of a step, in normal walking conditions, permits the dynamic analysis of the foot behavior. This introduction of the time dimension augments the potential of this type of clinical examination as an auxiliary tool for diagnostics and therapy planning [12].

The basic pedobarography system consists of a transparent plate trans-illuminated through its borders in such a way that the light is internally reflected. The plate is covered on its top by a single or dual thin layer of soft porous plastic material where the pressure is applied (see Figure 1).

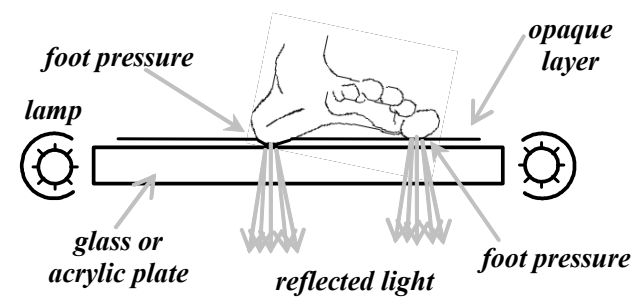

Figure 1: Basic (pedo)barography principle.

When observed from below, in the absence of applied pressure the plate is dark. However, when pressure is applied on top of the plastic layer, the plate displays bright areas that correspond to the light crossing the plate after reflection on the plastic layer. This reflection occurs due to the alteration of the local relation of light refraction indices resulting from the depletion of the air interface between the glass plate and the plastic layer. A good choice of materials and an adequate calibration of the image acquisition system, allow a nearly proportional relation between the local pressure and the observed brightness.

Using a practical setup as the one represented in Figure 2, a time sequence of pressure images can be captured. Figure 3 shows thirteen images of a captured sample sequence; as can be verified, the image data is 
very dense, as opposed to other measuring methods, and very rich in terms of the information it conveys on the interaction between the foot sole and the flat plate. Video 1 shows the image sequence considered.

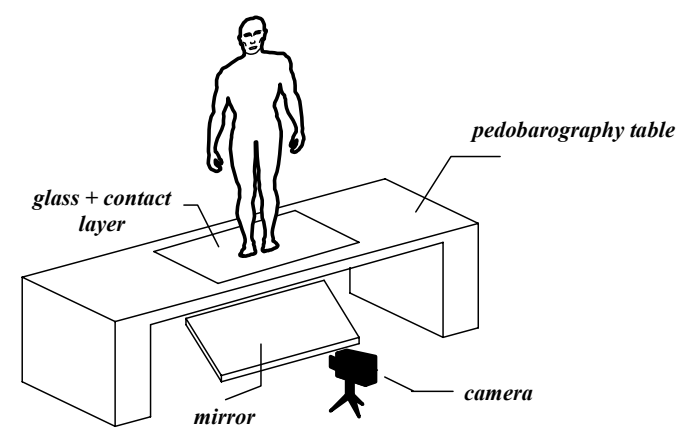

Figure 2: Basic setup of a pedobarography system.

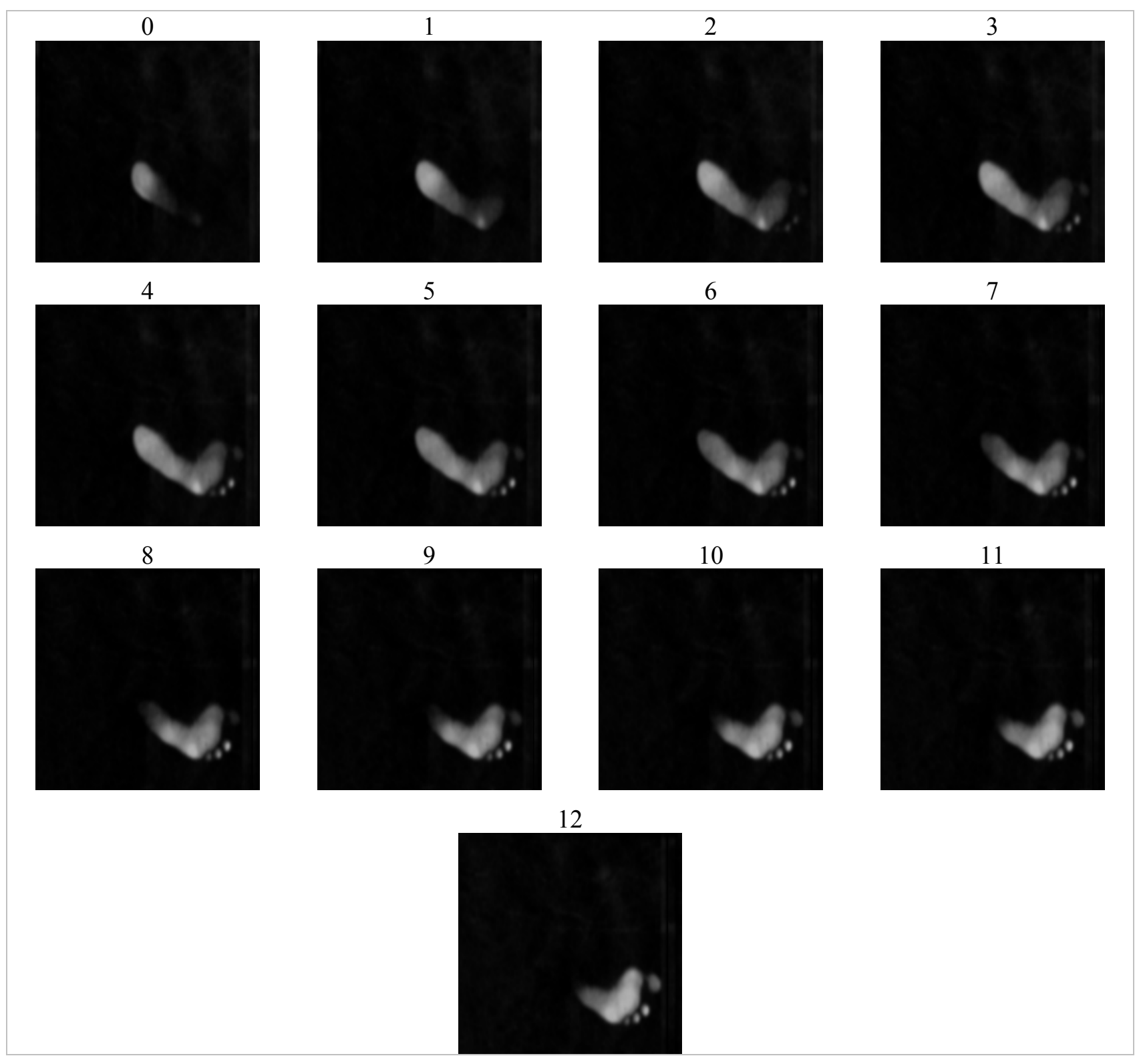

Figure 3: Example of a captured sequence composed by thirteen images. 


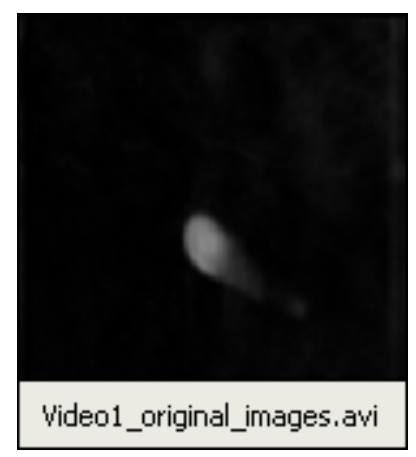

Video 1: Animation of the thirteen images of the sample sequence (click to see the video).

\section{Object Models}

In the initial stages of the work, the object contours in each image were extracted and the matching process was oriented to the contours' pixels [2], [13]. A practical difficulty arising from this approach is the possible existence of more than one contour for the object represented in each image (i. e. see Figure 7). To find the correspondence between each contours pair along the image sequence, two possible solutions were considered: i) use of a Kalman filtering (see [13], for example) approach to estimate and track the location of the contours' centroids along the image sequence; ii) use of a measure of the deformation energy necessary to align each contour pairs, selecting the lower energy pairs.

However, an additional problem is still present: the possibility that along the image sequence various contours will merge or split. In order to accommodate this possibility, a new model has been developed, similar to the one used in various applications working with controlled environment, such as in face analysis and recognition [14], [15]: The brightness level of each pixel is considered as the third coordinate of a 3D surface point. The resulting single surface model solves the two aforementioned problems.

The use of the surface model, also simplifies the consideration of isobaric contours, which are important in pedobarographic analysis, either for matching contours of equal pressure along the time sequence or for matching contours of different pressure in a single image [12], [13].

The following sections describe the object models used in this work and briefly describe their construction. Each model has its own advantages and shortcomings; for every particular problem, the best choice must be made [12], [13].

\subsection{Contour Model}

To determine the correspondence among two contours were used two modeling approaches:

- For each contour is used a single 2D Sclaroff's isoparametric finite element. In building this type of element, no previous ordering of the data points is required and Gaussian functions are used as interpolations functions. The method to determine the mass and stiffness matrices for this $2 \mathrm{D}$ element is described in, for example, [1].

- For each contour is built a finite elements model using linear axial finite elements (Figure 4). For this type of discretisation a previous ordering of the contour data points is required. The matrix formulation for these finite elements can be found in [16], for example. 


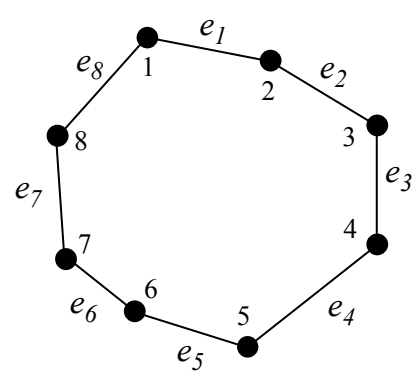

Figure 4: Modeling a contour by using a set $e_{i}$ of axial finite elements.

To determine in each image the contour pixels, are used standard image processing and analysis techniques; namely, thresholding, edge enhancement, hysteresis line detection and tracking [2]. For example, Figures 6 and 7 show the intermediate result and the final contours obtained from the image of Figure 5.

The sampled contours obtained from the thirteen images shown in Figure 3 are presented in Figure 8.

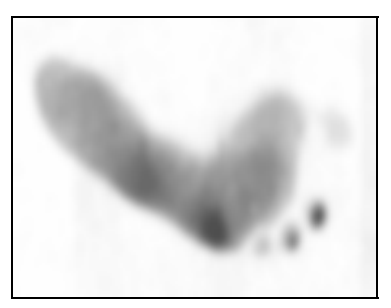

Figure 5: Image (negated) where contours must be found.

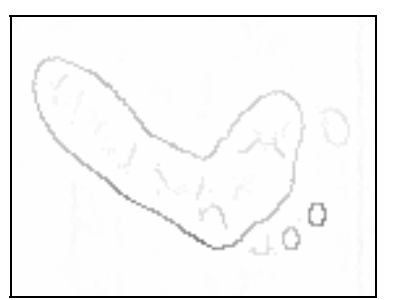

Figure 6: Result image after edge enhancement.

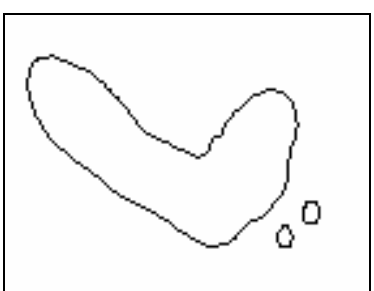

Figure 7: Contours obtained by a line detection and tracking algorithm [2].

\subsection{Surface Model}

For the surface model, two approaches were also considered:

- A single 3D Sclaroff's isoparametric finite element is used for each surface to be matched. Again, it must be noticed that there is no requirement for previous ordering of the surface nodes. The matrix building for these finite elements can be found in [1].

- Each surface model is built by using linear axial finite elements (Figure 9). The previous ordering of the surface data points is required. The matrix formulation for these finite elements can be found in [16], for example.

The used methodology to determine the data points of each surface can be summarized as follows:

1. Noise pixels (that is, pixels with brightness lower than a calibration threshold level) are removed and a Gaussian-shaped smoothing filter is applied to the image (Figure 10);

2. The circumscribing rectangle of the object to be modeled is determined and the image is sampled within that area (Figure 11);

3. A 2D Delaunay triangulation (see, for example, $[25,26]$ ) is performed on the sampled points, using the point brightness as the third coordinate;

4. In order to reduce the number of nodes used, and thus the computational cost, the triangular mesh is simplified using a decimation algorithm (see, for example, $[25,26]$ );

5. To reduce the high frequency noise associated to the mesh, is used a Laplacian smoothing algorithm (see, for example, $[25,26])$;

6. Finally, in order to have similar ranges of values in all coordinates, a scale change is performed on the third coordinate (derived from brightness) (Figure 12). 


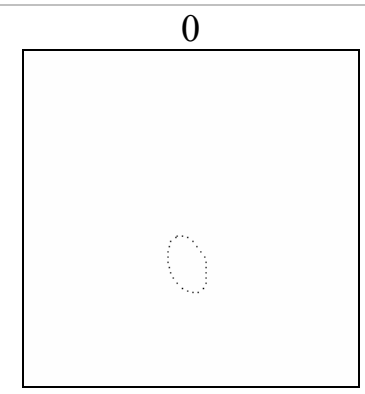

4

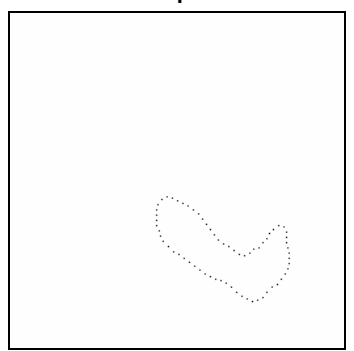

8

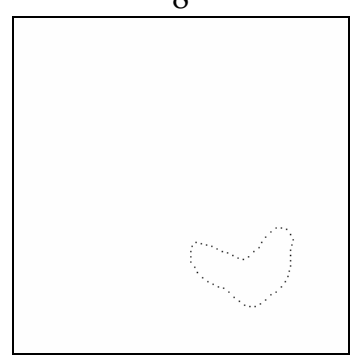

1

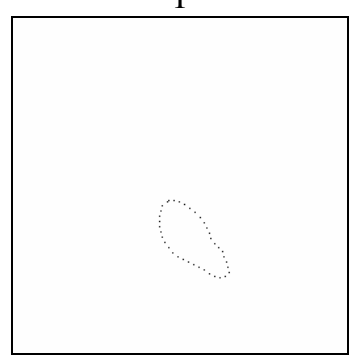

5

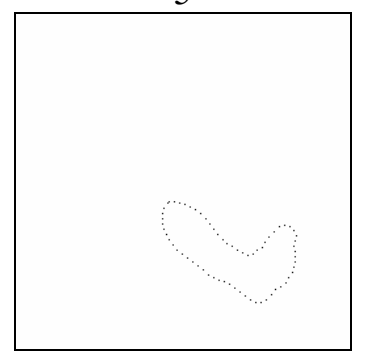

9

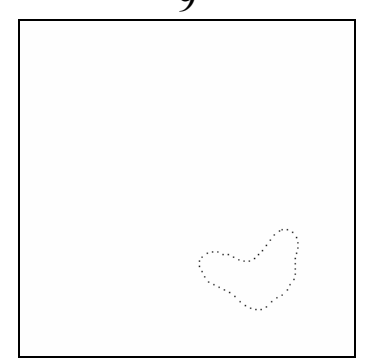

2

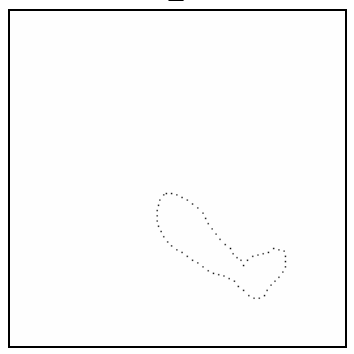

6

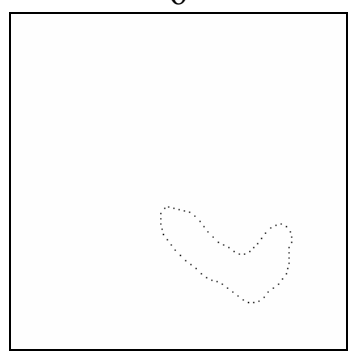

10

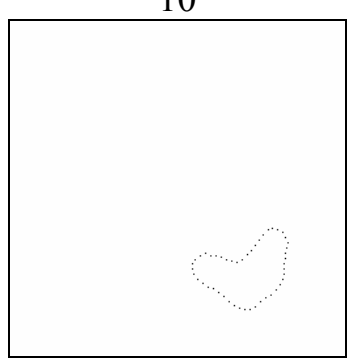

3

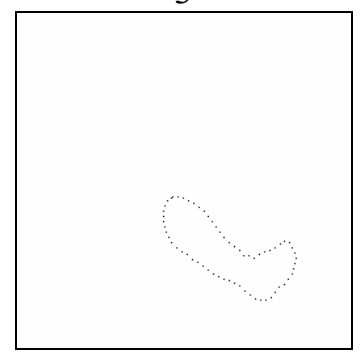

7

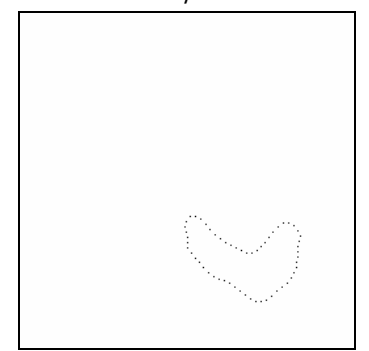

11

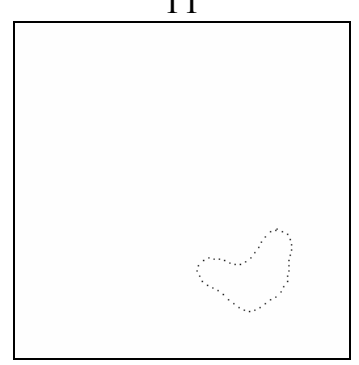

12

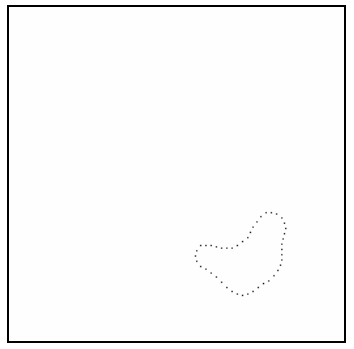

Figure 8: Sampled contours obtained from the original image sequence of Figure 3.

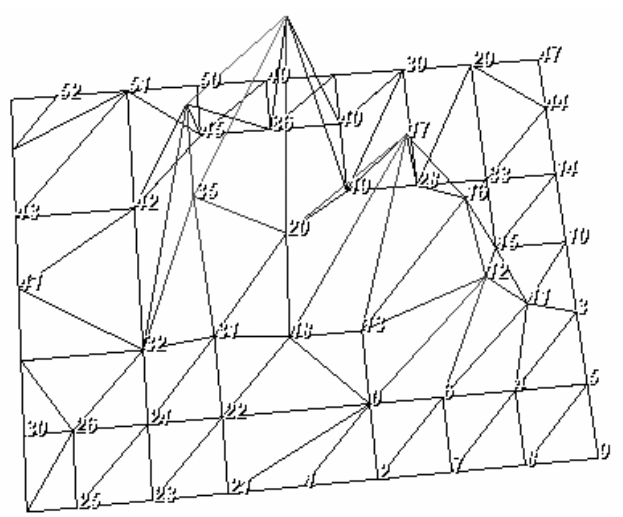

Figure 9: Modeling a surface by using a set of axial finite elements. (Each node is connected to its neighbors through axial elements.) 


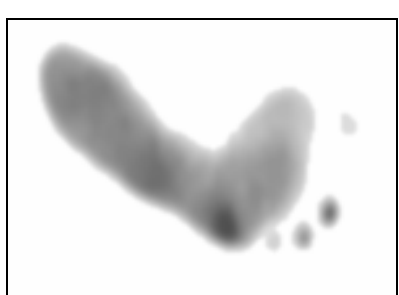

Figure 10: Image (negated) after noise removal and Gaussian filtering.

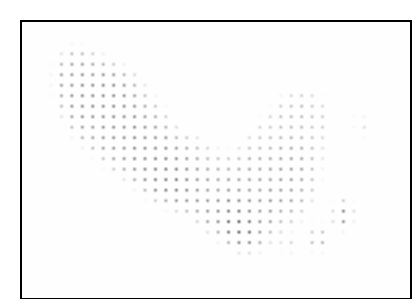

Figure 11: Object sampling.

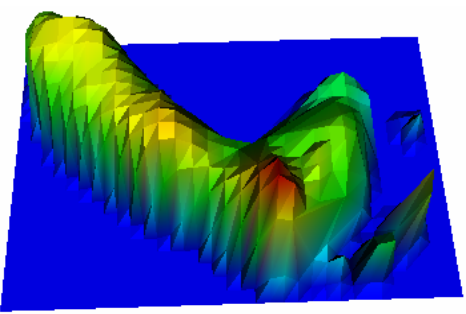

Figure 12: Resulting surface.

The surfaces obtained from the original images presented in Figure 3 are visible in Figure 13. The original images with identification (ID) 0 (zero) and 1 (one) were not considered.

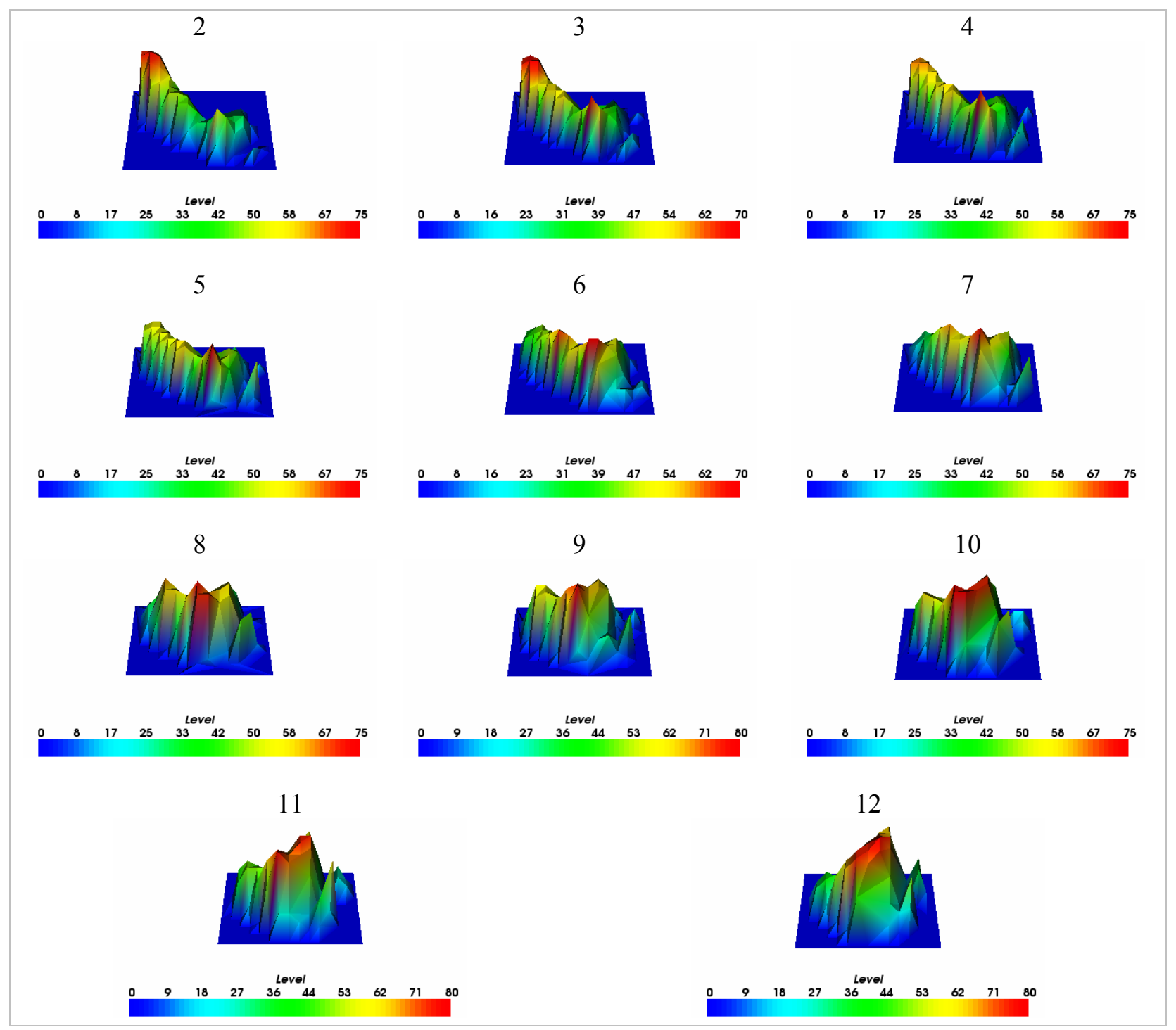

Figure 13: Surfaces obtained from the last eleven images of the example sequence.

\subsection{Isobaric Contour Model}

As in the two previous models, the approaches used to match isobaric contours are: 
- A single Sclaroff's isoparametric finite element, either 2D or 3D, is used to model each contour.

- A set of linear axial finite elements are used to build each contour model.

The isobaric contours are extracted from the correspondent surface, beforehand obtained using the procedures described in the previous section (Figure 14).

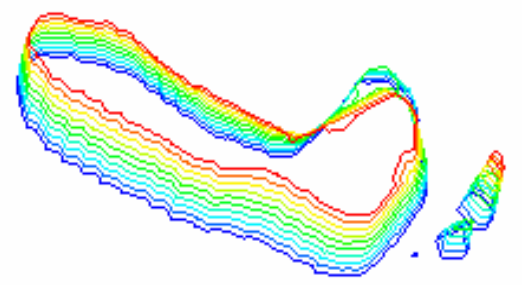

Figure 14: Ten isobaric contours extracted from the surface of figure 12 .

\section{Matching Methodology}

Figure 15 displays a diagram of the adopted physical matching methodology. The locations of the objects data points in each image, $X=\left[X_{1} \ldots X_{m}\right]$, are used as the nodes of a finite elements model made of an elastic material. Next, the eigenmodes $\{\phi\}_{i}$ of the model are computed, providing an orthogonal description of the object and its natural deformations, ordered by frequency. Using a matrix based notation, the eigenvectors matrix $[\Phi]$ and the eigenvalues diagonal matrix $[\Omega]$ can be written as in equation (1) for $2 \mathrm{D}$ objects and as in equation (2) for 3D objects. The eigenvectors, also called shape vectors [1], [2], [16], [17], describe how each vibration mode deforms the object by changing the original data point locations: $X_{\text {deformed }}=X+a\{\phi\}_{i}$.

$$
\begin{aligned}
& {[\Phi]=\left[\{\phi\}_{1}|\cdots|\{\phi\}_{2 m}\right]=\left[\begin{array}{c}
\{u\}_{1}^{T} \\
\vdots \\
\{u\}_{m}^{T} \\
\{v\}_{1}^{T} \\
\vdots \\
\{v\}_{m}^{T}
\end{array}\right] \text { and }[\Omega]=\left[\begin{array}{ccc}
\omega_{1}^{2} & & 0 \\
& \ddots & \\
0 & & \omega_{2 m}^{2}
\end{array}\right] \text {, }} \\
& {[\Phi]=\left[\{\phi\}_{1}|\cdots|\{\phi\}_{3 m}\right]=\left[\begin{array}{c}
\{u\}_{1}^{T} \\
\vdots \\
\{u\}_{m}^{T} \\
\{v\}_{1}^{T} \\
\vdots \\
\{v\}_{m}^{T} \\
\{w\}_{1}^{T} \\
\vdots \\
\{w\}_{m}^{T}
\end{array}\right] \text { and }[\Omega]=\left[\begin{array}{ccc}
\omega_{1}^{2} & & 0 \\
& \ddots & \\
0 & & \omega_{3 m}^{2}
\end{array}\right] \text {. }}
\end{aligned}
$$

The first three (in 2D) or six (in 3D) vibration modes are the rigid body modes of translation and rotation; the remaining modes are non-rigid [1], [2], [16], [17]. In general, lower frequency modes describe global deformations, while higher frequency modes essentially describe local deformations. This type of ordering, from global to local behaviour, is quite useful for object matching and recognition. 


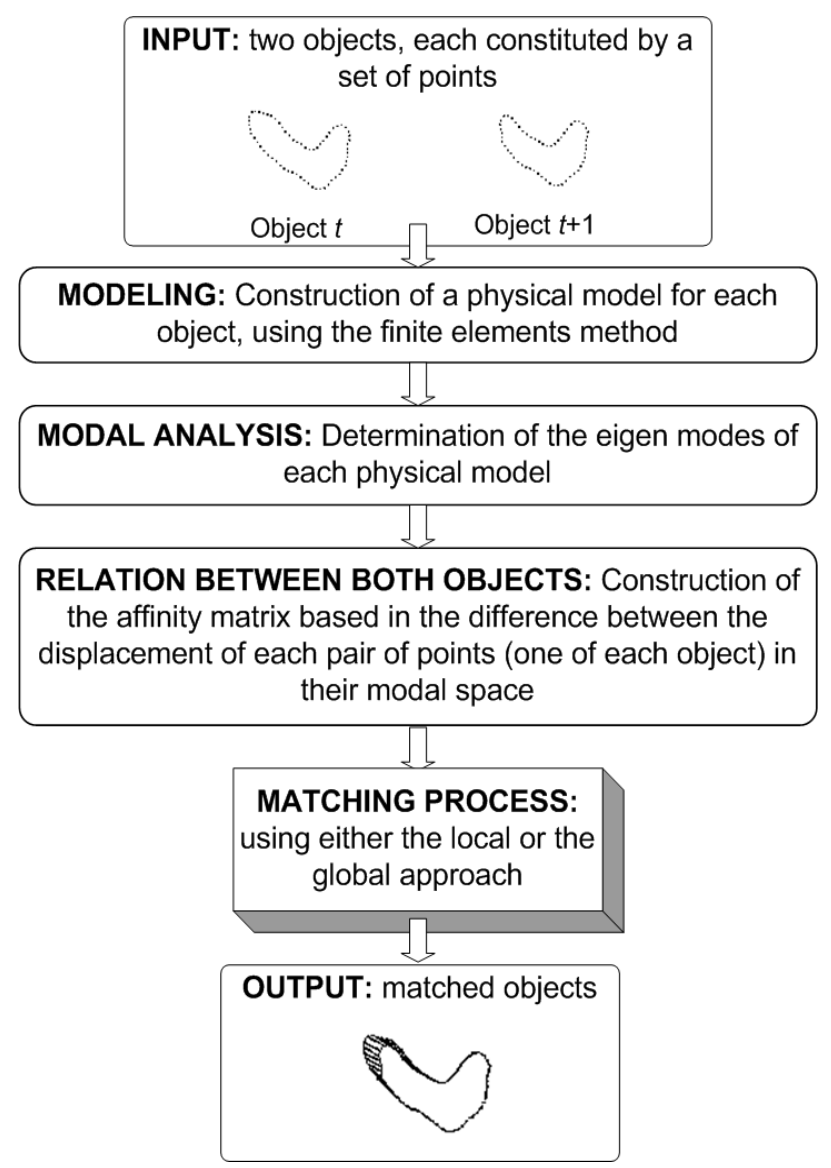

Figure 15: Diagram of the adopted matching methodology.

The eigenmodes also form an orthogonal, object-centred coordinate system for the location of the data points, i.e., the location of each point is uniquely described in terms of each eigenmode displacement. The transformation between the Cartesian image coordinates and the modal coordinates system is achieved through the eigenvectors matrix of the physical model.

Two sets of data points, for example, corresponding to the objects represented in two different images of a sequence, are to be compared in the modal eigenspace. The main idea is that the low order modes of two similar objects will be very close, even in the presence of an affine transformation, a non-rigid deformation, a local shape variation, or noise.

Using the above concept, data correspondence is obtained by modal matching. In this work, two matching search procedures are considered: (i) a local search or (ii) a global search. Both search strategies consider an affinity matrix $[Z]$, constructed from the Euclidian distance between the characteristic vectors of each physical model, whose elements are, for $2 \mathrm{D}$ and for 3D, respectively:

$$
\begin{aligned}
& Z_{i j}=\left\|\{u\}_{t, i}-\{u\}_{t+1, j}\right\|^{2}+\left\|\{v\}_{t, i}-\{v\}_{t+1, j}\right\|^{2}, \\
& Z_{i j}=\left\|\{u\}_{t, i}-\{u\}_{t+1, j}\right\|^{2}+\left\|\{v\}_{t, i}-\{v\}_{t+1, j}\right\|^{2}+\left\|\{w\}_{t, i}-\{w\}_{t+1, j}\right\|^{2} .
\end{aligned}
$$

The local search strategy was proposed in the original modal matching methodology [1], [2], [13], and basically it consists in seeking each row of the affinity matrix for its lowest value, considering the associated correspondence if, and only if, that value is also the lowest of the related column. With this search philosophy, the models nodes are considered as independent entities and so the original object structure is ignore. 
As previously referred, the matching problem considering only matches of type "one to one" and objects with equal number of data points can be considered as a classical assignment problem, which is a particular case of an optimization problem [18], [20]. In the formulation of this type of problem is assumed that: the number of data points of both objects is the same, $n$; it is known the assignment cost, $Z_{i j}$, of each pair of points $(i, j)$, where $i$ is a point of object $t$ and $j$ is a point of object $t+1$. As the notation tries to evidence, this assignment cost is equal to the element in line $i$ and column $j$ of the affinity matrix, $[Z]$.

The assignment problem initially appeared to mathematically formulate problems in which $n$ jobs/works must be distributed by $n$ tasks, with the restriction that each job/work had to be assigned to one, and only one, task and vice-versa, subject to the minimization of the global assignment cost. In this work, the jobs/works are the data points of the object $t$ and the tasks are the data points of the object $t+1$. So, for the mathematical formulation of this matching problem, let's consider:

$$
x_{i j}=\left\{\begin{array}{cc}
1 & \text { if point } i \text { of } t \text { is assigned to point } j \text { of } t+1 \\
0 & \text { otherwise }
\end{array},\right.
$$

with $i, j=1,2, \ldots, n$. Next expressions follow the typical structure of a mathematical programming problem, with an objective function and a set of restrictions in the problem's variables:

$$
\begin{aligned}
& \text { minimize } f=\sum_{i=1}^{n} \sum_{j=1}^{n} Z_{i j} x_{i j}, \\
& \text { subject to } \sum_{j=1}^{n} x_{i j}=1 \text {, with } i=1,2, \ldots, n, \\
& \sum_{i=1}^{n} x_{i j}=1, \text { with } j=1,2, \ldots, n, \\
& \text { and } x_{i j} \in\{0,1\}, \forall i, j .
\end{aligned}
$$

In equation (6), the function $f$ takes the value of the assignment total cost. The first restriction (7), forces each data point in object $t$ to be assigned to one, and only one, data point in object $t+1$. The second restriction (8), forces each data point in object $t+1$ to be assigned to one, and only one, data point in object $t$. The third and last restriction (9), forces the problem's variables, $x_{i j}(i, j=1,2, \ldots, n)$, to take one of the two possible values $\{0,1\}$.

To solve the assignment (matching) problem we considered three algorithms: the Hungarian method [18], [19]; the Simplex method for flow problems [18], [21]; and the LAPm algorithm [18], [22]. The Hungarian method is the most well known method for the resolution of the assignment problem. The Simplex method for flow problems solves problems with less rigid restrictions than the assignment problem, but where this last can be included as a special case. The LAPm method is a considerably recent algorithm developed to solve classical assignment problems.

Once the optimal global matching solution is found, as in the original local matching procedure, the matches that exceed a pre-established matching threshold level are eliminated from that solution. The relevance of this restriction is higher with this global matching approach, because a solution of the assignment problem always has the maximum number of matches of type "one to one", due to the problem's restrictions.

Case the number of data points of the two objects to be matches are different, with the usual matching restriction that allows only matches of type "one to one", will necessarily exist data points that won't be matched. The solution found was initially add fictitious points to the model with fewer data points; this way, we solve the optimization problem requirement of the affinity matrix be necessarily square. Then, after the 
optimization phase, we have some real objects data points, the excess points, matched with the fictitious elements previously add. Finally, these excess points are matched adequately with real objects data points, using a neighbourhood and an affinity criterion, as follows:

- For each excess point, the developed algorithm fits it between its matched nearest neighbours;

- From the correspondences of those neighbours in the other object, it is determined the best correspondence for the excess point, minimizing the costs and considering that the neighbours must remain as so and that there mustn't exist crossed matches;

- As in the optimization phase, the obtained matches will only be considered as good matches if, and only if, the pre-established matching threshold level is respected.

With this solution, for the excess objects data points are allowed matches of type "one to many" or vice versa.

Note that, for all the used methodologies in the matching framework it's not considered any additional information about the original image sequence neither about the represented objects.

\section{Results}

The presented matching methodology was integrated in a generic software platform for deformable objects [2], [23], previous developed using Microsoft Visual $\mathrm{C}^{++}$, the Newmat [24] library for matrix computation and the VTK - The Visualization Toolkit [25], [26] for 3D visualization, mesh triangulation, simplification and smoothing, and for isobaric contours extraction.

This section presents some experimental results obtained on objects extracted from dynamic pedobarography images, using the adopted matching methodology. First, are presented results considering contour, then surface, and finally isocontour models. In all cases, the object pairs considered were just selected for example purposes.

All the results presented in this section, were obtained considering in the objects modeling the Sclaroff's isoparametric finite element and rubber as the virtual elastic material, and using $25 \%$ of the models vibration modes in the matching phase.

\subsection{Contour object matching}

Considering the pairs of contours with ID 2/3, 3/4 and 10/11, previously presented in Figure 8, using the matching methodology adopted, we obtain the matches shown in Figures 16, 17, and 18, respectively. In these figures, the contours data points, and also the matched pairs, are connected for better visualization.

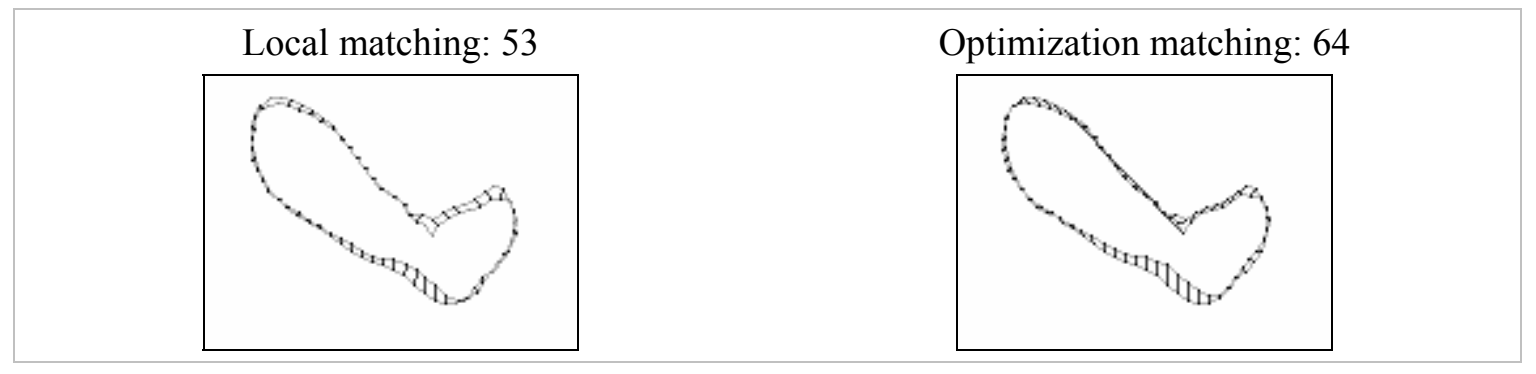

Figure 16: Matches obtained between contours with ID 2 (64 nodes) and 3 (64 nodes).

The matches found between the contours extracted from the last eleven images (ID 2 to 12) of the example sequence, are present in Figure 19. With Videos 2, 3 and 4 it is possible to see these matches along the image sequence. The number and the percentage of matches obtained during the same sequence are indicated in Table 1.

The results obtained using the local search strategy, were in range of $50.2 \%$ to $82.8 \%$ and present all good quality; instead, the optimization search procedure had always $100 \%$ of matching success, and in generally the found matches also have good quality and the excess nodes were reasonably matched. 
One important remark is the use of the same methodology parameters for all matching cases. If the parameters were adjusted for each contour pair, the results could be improved. In the same way, the matching threshold level was chosen to allow all the established matches, avoiding the rejection of the less adequate matches (see Figure 18, for example).

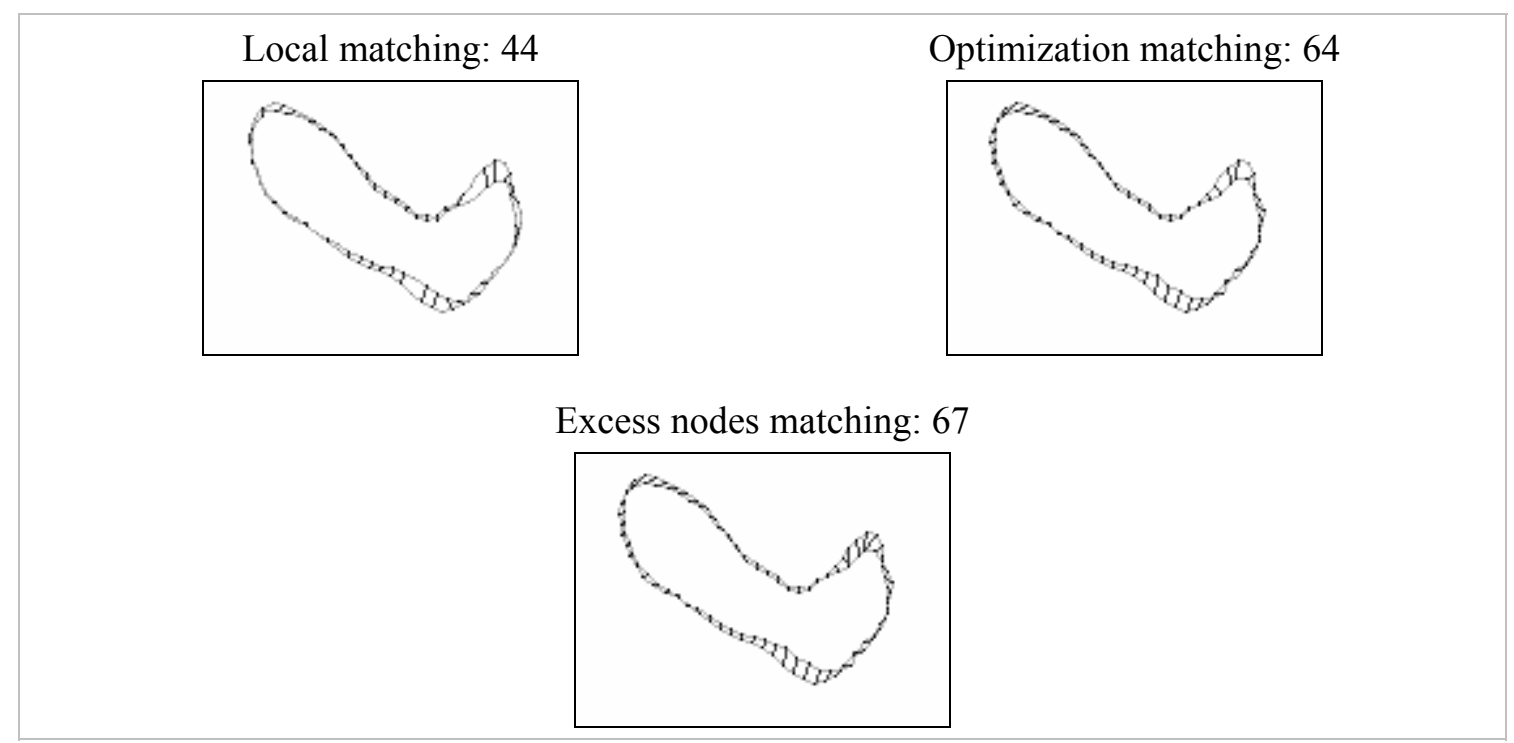

Figure 17: Matches obtained between contours with ID 3 (64 nodes) and 4 (67 nodes).

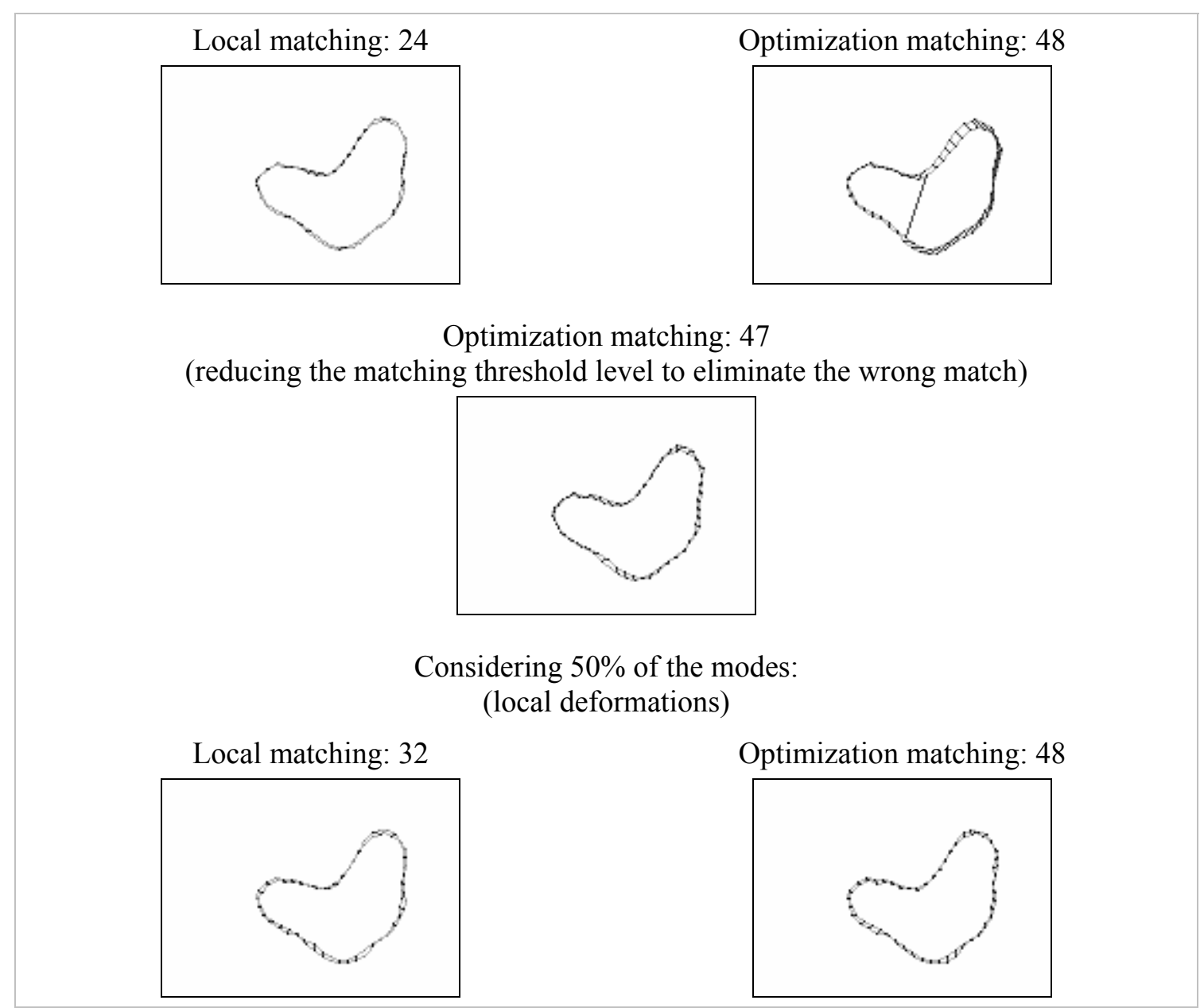

Figure 18: Matches obtained between contours with ID 10 (48 nodes) and 11 (48 nodes). 


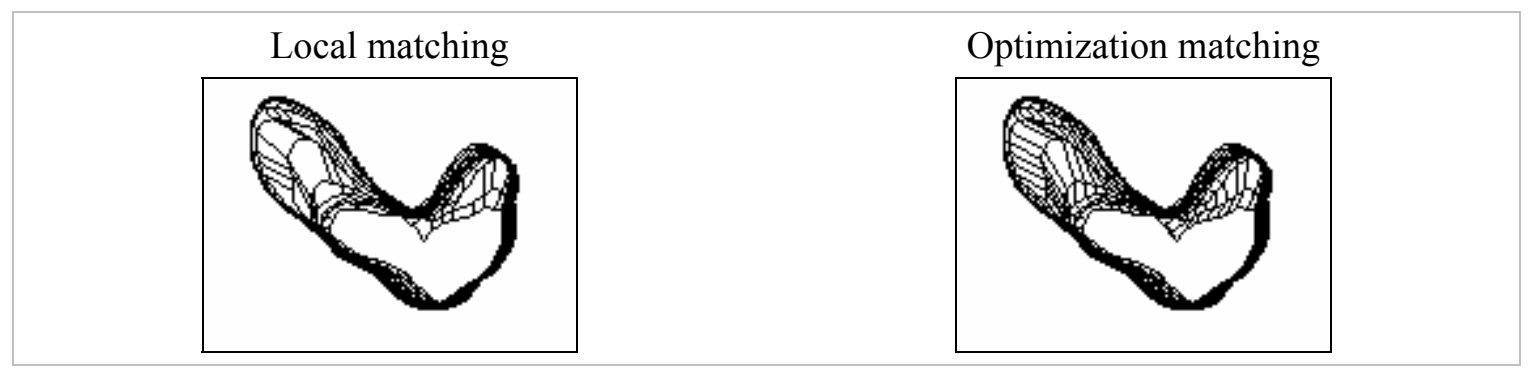

Figure 19: Matches obtained between all eleven contours (from ID 2 to 12).

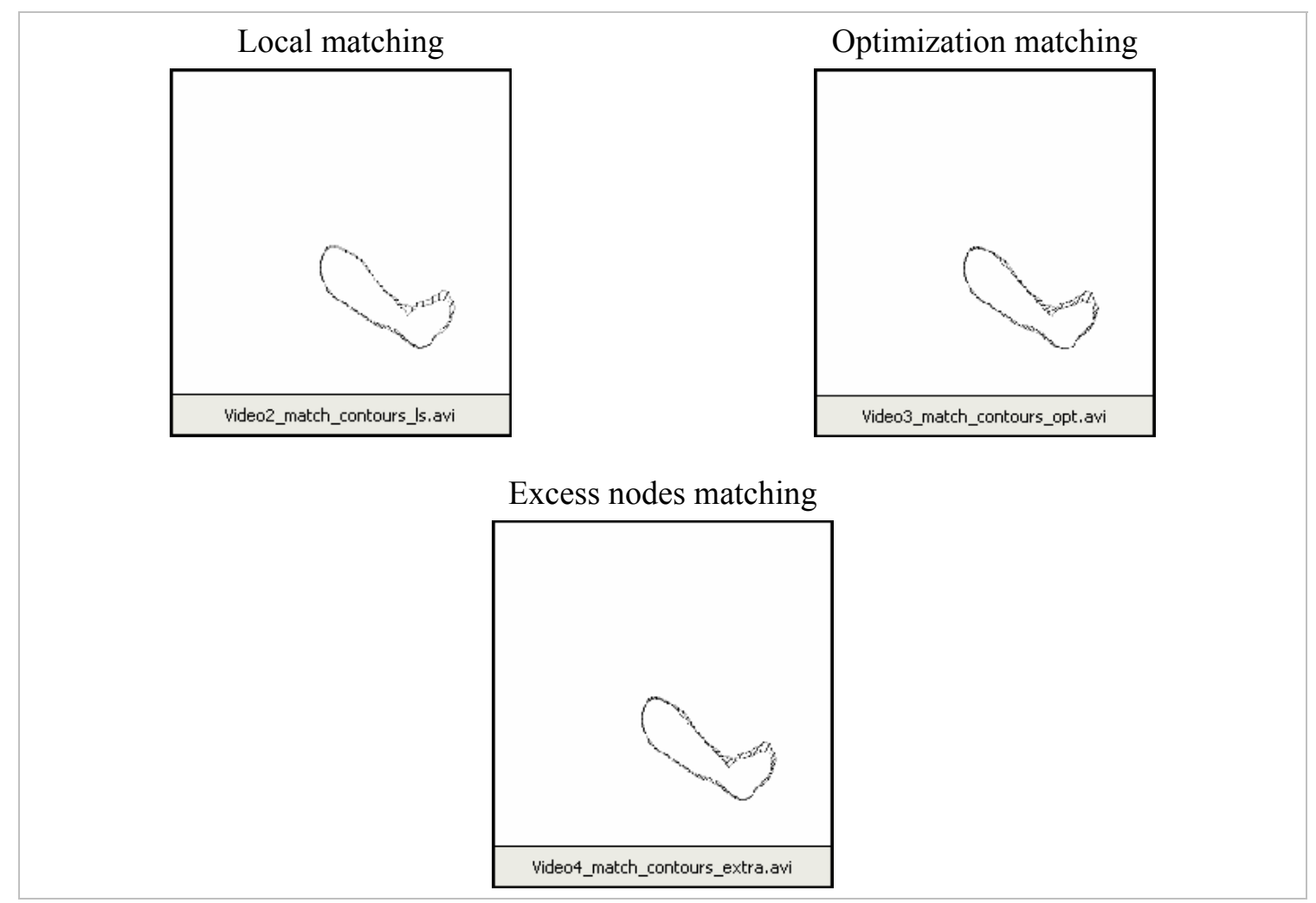

Videos 2, 3 and 4: Matches found between the contours extracted from the last eleven images of the example sequence (click to see the video).

\begin{tabular}{||c|c|c|c|c|c|c||}
\cline { 3 - 7 } \multicolumn{2}{c|}{} & \multicolumn{2}{c|}{ Local } & \multicolumn{2}{c||}{ Optimization } & Excess Nodes \\
\hline \hline contours ID & $\boldsymbol{N}^{\boldsymbol{o}}$ Nodes & $\boldsymbol{N}^{\boldsymbol{o}}$ Match & $\%$ Match & $\boldsymbol{N}^{\boldsymbol{o}}$ Match & $\%$ Match & $\boldsymbol{N}^{\boldsymbol{o}}$ Match \\
\hline 2,3 & $64 / 64$ & 53 & $82,8 \%$ & 64 & $100 \%$ & \\
\hline 3,4 & $64 / 67$ & 44 & $68,8 \%$ & 64 & $100 \%$ & 67 \\
\hline 4,5 & $67 / 67$ & 55 & $82,1 \%$ & 67 & $100 \%$ & \\
\hline 5,6 & $67 / 64$ & 47 & $73,4 \%$ & 64 & $100 \%$ & 67 \\
\hline 6,7 & $64 / 58$ & 46 & $79,3 \%$ & 58 & $100 \%$ & 64 \\
\hline 7,8 & $58 / 51$ & 30 & $58,8 \%$ & 51 & $100 \%$ & 58 \\
\hline 8,9 & $51 / 50$ & 33 & $66,0 \%$ & 50 & $100 \%$ & 51 \\
\hline 9,10 & $50 / 48$ & 26 & $54,2 \%$ & 48 & $100 \%$ & 50 \\
\hline 10,11 & $48 / 48$ & 24 & $50,0 \%$ & 48 & $100 \%$ & \\
\hline 11,12 & $48 / 46$ & 25 & $54,3 \%$ & 46 & $100 \%$ & 48 \\
\hline
\end{tabular}

Table 1: Number ( $\mathrm{N}^{\circ}$ Match) and percentage of matches (\% Match) obtained between the contours extracted from the last eleven images (ID 2 to 12) of the example sequence. 


\section{2 - Surface matching}

The matches found between the surfaces models with ID 2/3, 4/5 and 11/12, each one build from the correspondent image of the example sequence, using the adopted matching methodology, are shown in the Figures 20, 21, and 22, respectively. In these figures, the matched nodes are connected for better viewing and two different views are presented.

The number and the percentage of matches obtained considering the surfaces models build from the last eleven images of the example sequence are indicated in Table 2.

The results of the local search strategy were in range of $33.3 \%$ to $87.8 \%$, and, attending to the high objects deformation, we can consider that the found matches have good quality. In other hand, the search strategy based on optimization techniques had always 100\%, and generally the matches present also good quality.

As in the contour case, all the parameters of the matching methodology were considered constant along the sequence.

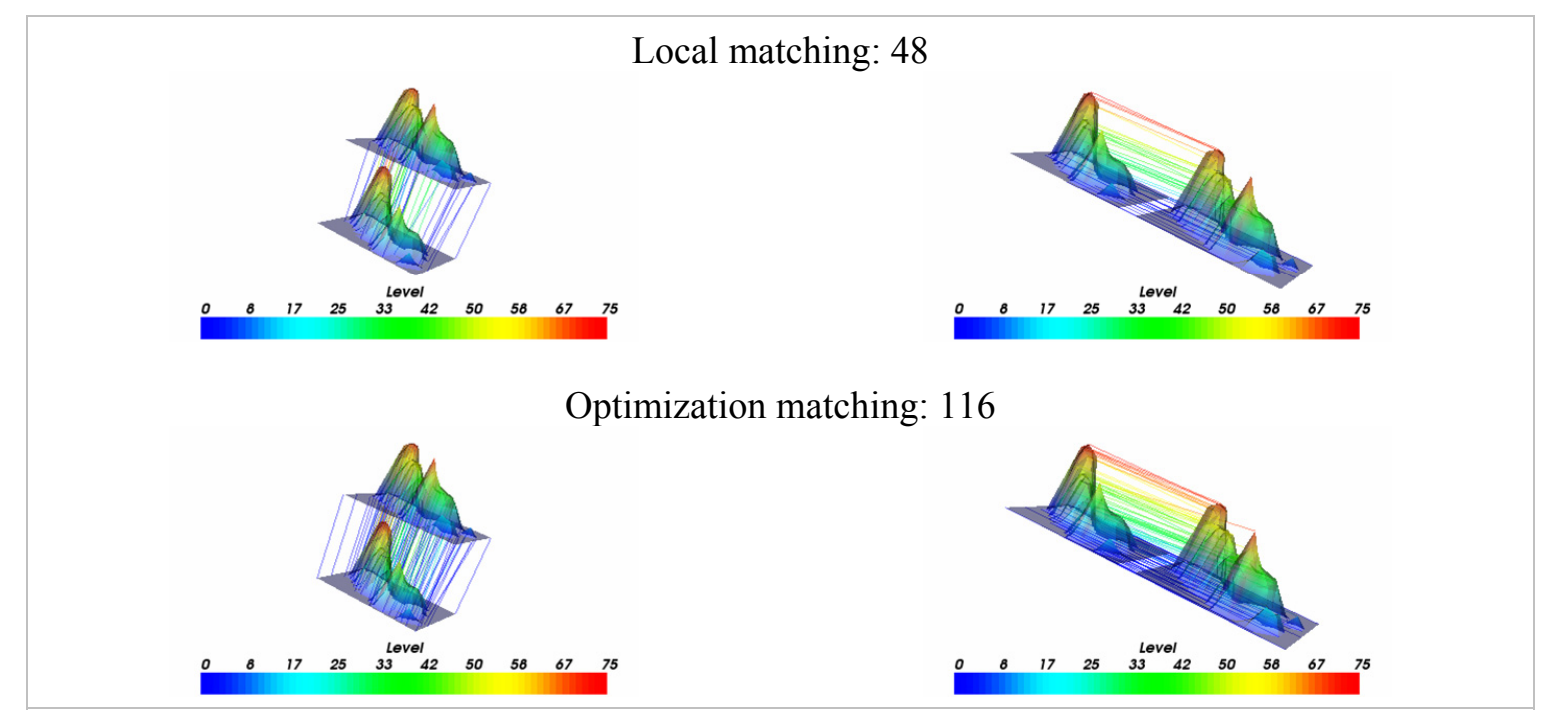

Figure 20: Matches obtained between surfaces with ID 2 (116 nodes) and 3 (131 nodes).

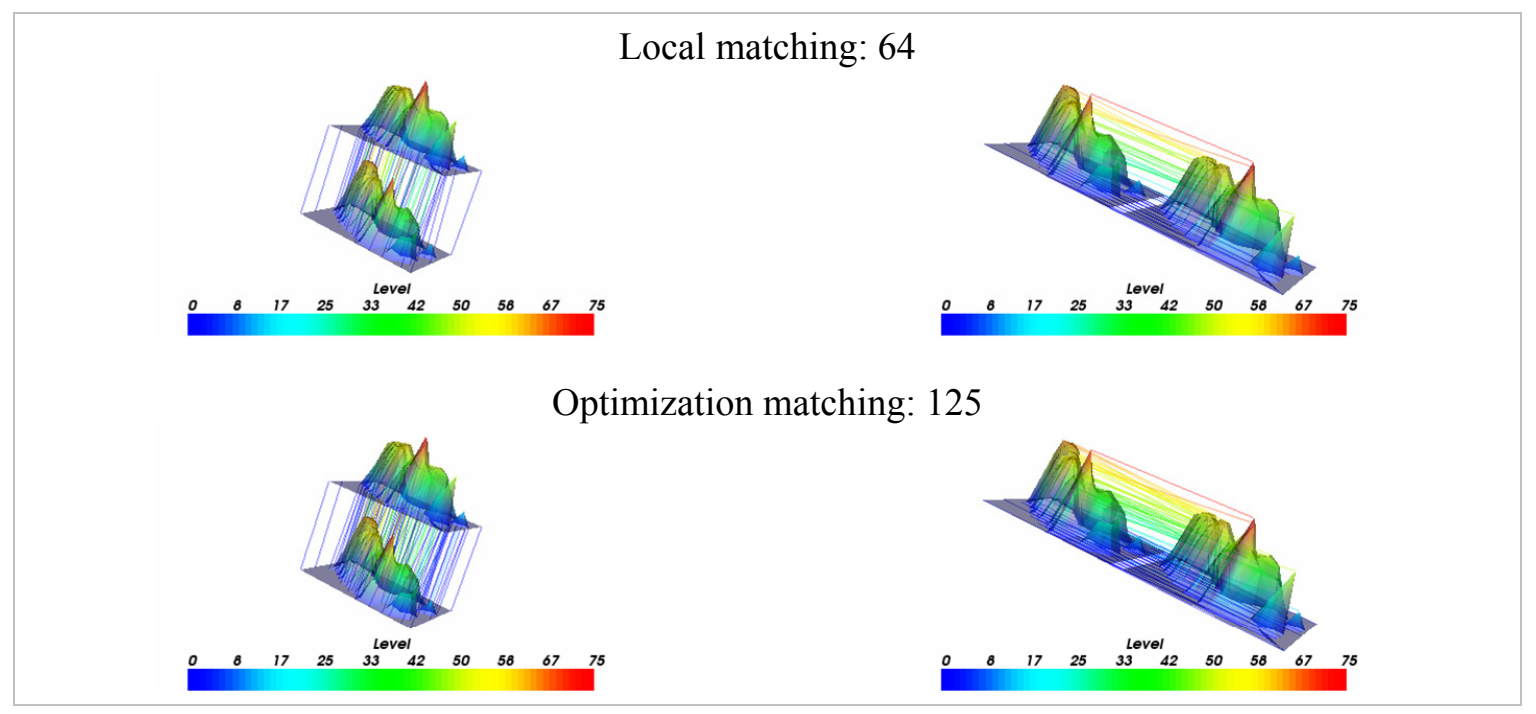

Figure 21: Matches obtained between surfaces with ID 4 (131 nodes) and 5 (125 nodes). 


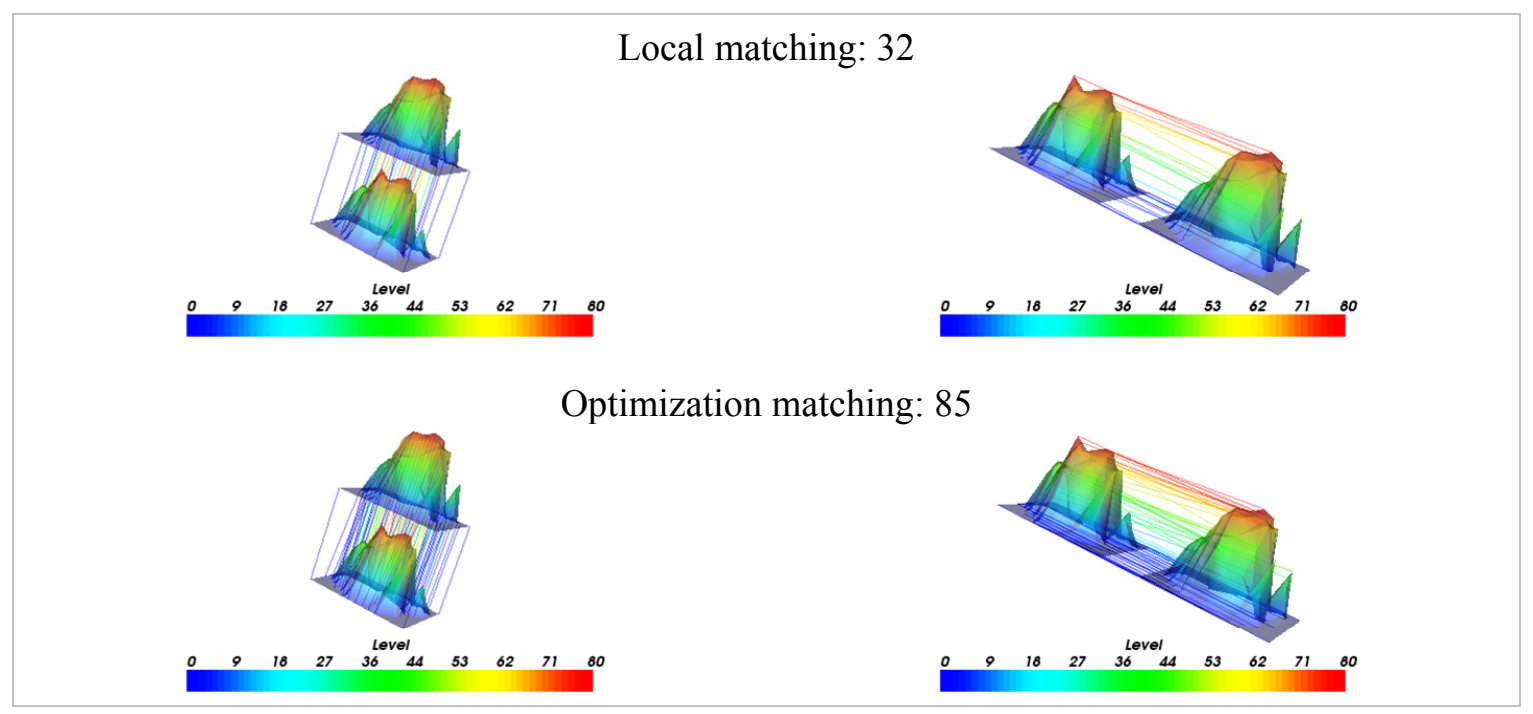

Figure 22: Matches obtained between surfaces with ID 11 (99 nodes) and 12 ( 85 nodes).

\begin{tabular}{||c|c|c|c|c|c||}
\cline { 4 - 6 } \multicolumn{2}{c|}{} & \multicolumn{2}{c|}{ Local } & \multicolumn{2}{c||}{ Optimization } \\
\hline \hline Surfaces ID & $\boldsymbol{N}^{\boldsymbol{o}}$ Nodes & $\boldsymbol{N}^{\boldsymbol{o}}$ Match & $\boldsymbol{\%}$ Match & $\boldsymbol{N}^{\boldsymbol{o}}$ Match & $\%$ Match \\
\hline 2,3 & $116 / 131$ & 48 & $41,3 \%$ & 116 & $100 \%$ \\
\hline 3,4 & $131 / 131$ & 115 & $87,8 \%$ & 131 & $100 \%$ \\
\hline 4,5 & $131 / 125$ & 64 & $51,2 \%$ & 125 & $100 \%$ \\
\hline 5,6 & $125 / 109$ & 67 & $61,5 \%$ & 109 & $100 \%$ \\
\hline 6,7 & $109 / 107$ & 51 & $47,7 \%$ & 107 & $100 \%$ \\
\hline 7,8 & $107 / 96$ & 32 & $33,3 \%$ & 96 & $100 \%$ \\
\hline 8,9 & $96 / 98$ & 52 & $54,1 \%$ & 96 & $100 \%$ \\
\hline 9,10 & $98 / 95$ & 56 & $58,9 \%$ & 95 & $100 \%$ \\
\hline 10,11 & $95 / 99$ & 52 & $54,7 \%$ & 95 & $100 \%$ \\
\hline 11,12 & $99 / 85$ & 32 & $37,4 \%$ & 85 & $100 \%$ \\
\hline \hline
\end{tabular}

Table 2: Number ( $\mathrm{N}^{\circ}$ Match) and percentage of matches (\% Match) obtained with the surfaces build from the last eleven images of the example sequence.

\subsection{Isocontour matching}

Using the proposed matching methodology to establish the correspondences between the isocontours with ID $1 / 2,4 / 5$, and $6 / 7$, all extracted from the image with ID 6 of the example sequence, were obtained the matches shown in Figures 23, 24, and 25, respectively. As in the surface case, in these figures the matched nodes are connected and two different views are presented.

The matches found considering eleven isocontours extracted from the same image (with ID 6) of the example sequence are shown in Figure 26. With Videos 5 and 6 it is possible to see these matches along the referred isocontour sequence.

The number and the percentage of the obtained matches considering the referred eleven isocontours are indicated in the Table 3.

Using the local search strategy, the results obtained were in range of $50.0 \%$ to $94.1 \%$, and the matches found could be considered of good quality. Instead, the search based on optimization techniques had always $100 \%$ of matching success, and in generally the matches found have also good quality and the excess nodes were reasonably matched.

As in the contour and surface cases, the matching results obtained could be improved if the parameters of the adopted methodology were adjusted for each isocontour pair. 


\section{Local matching: 52}

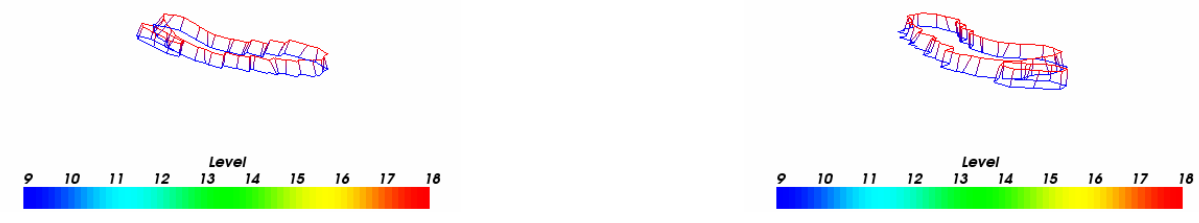

Optimization matching: 76
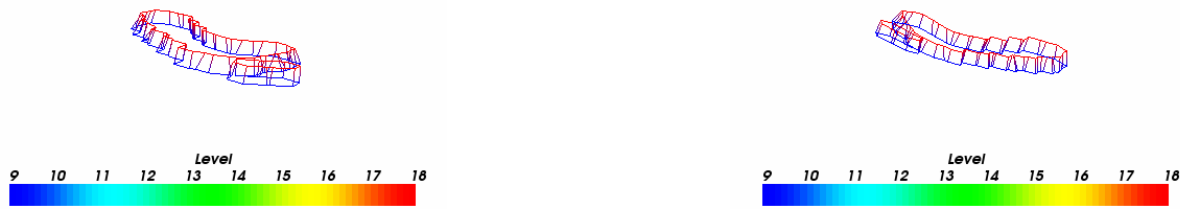

Figure 23: Matches obtained between isocontours with ID 1 (76 nodes) and 2 (76 nodes).

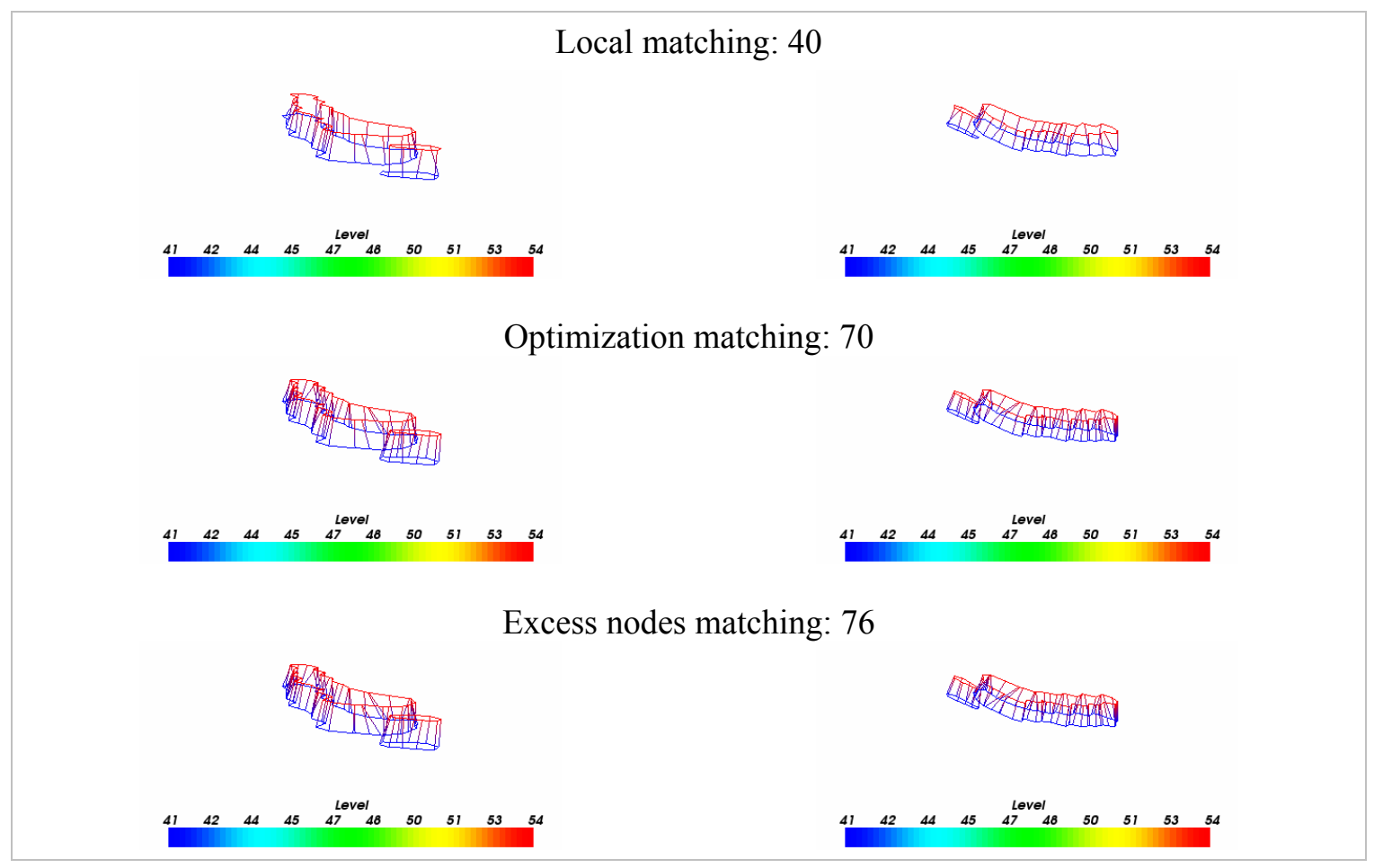

Figure 24: Matches obtained between isocontours with ID 4 (76 nodes) and 5 (70 nodes).

\section{Conclusions}

The several experimental tests carried through this work, some reported in this paper, allow the presentation of some observations and conclusions.

The physical methodology proposed, for the determination of correspondences between two objects, using optimization techniques on the matching phase, when compared with the one previously developed that considers local search, obtained always an equal or higher number of satisfactory matches. It was also verified that the number of matches found is independent from the optimization algorithm considered.

In some experimental cases, in order to obtain a higher number of satisfactory matches using the local search strategy, the parameters of the physical methodology had to be carefully chosen. In those same cases, the application of the optimization strategy in the matching phase, beyond the good quality of the matches found, revealed less sensible to the methodology parameters. This suggests that using the proposed global 
search strategy in the matching phase, the adopted physical methodology become easier to handle and also more adjustable to different kinds of applications.

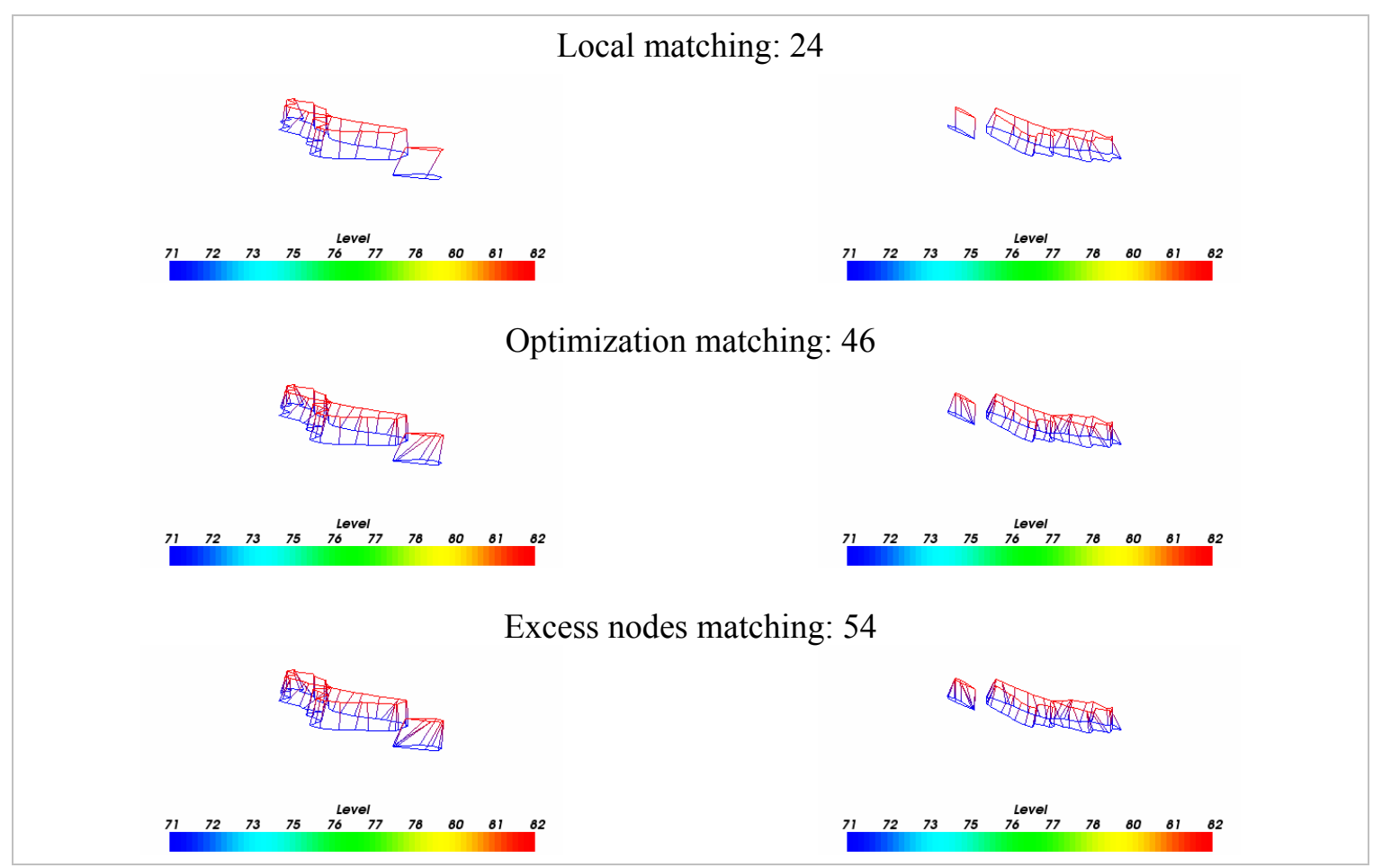

Figure 25: Matches obtained between isocontours with ID 6 (54 nodes) and 7 (46 nodes).

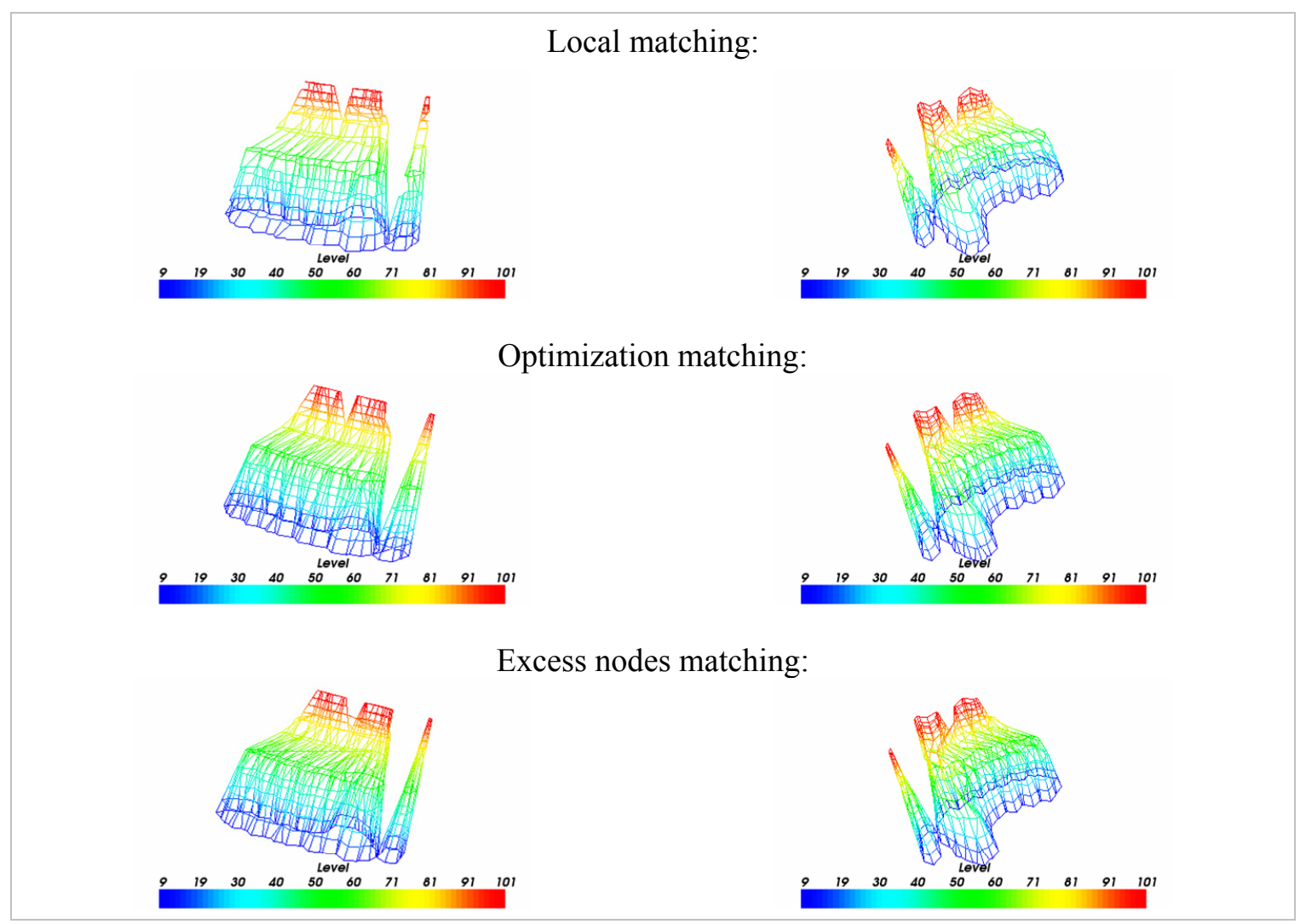

Figure 26: Matches obtained between the eleven isocontours extracted from the image with ID 6 of the example sequence. 


\begin{tabular}{||c|c|c|c|c|c|c||}
\cline { 3 - 7 } \multicolumn{2}{c|}{} & \multicolumn{2}{c|}{ Local } & \multicolumn{2}{c|}{ Optimization } & Excess Nodes \\
\hline \hline Isocontours ID & $\boldsymbol{N}^{\boldsymbol{o}}$ Nodes & $\boldsymbol{N}^{\boldsymbol{o}}$ Match & $\boldsymbol{\%}$ Match & $\boldsymbol{N}^{\boldsymbol{o}}$ Match & \% Match & $\boldsymbol{N}^{\boldsymbol{o}}$ Match \\
\hline 1,2 & $76 / 76$ & 52 & $68,4 \%$ & 76 & $100 \%$ & \\
\hline 2,3 & $76 / 74$ & 58 & $78,4 \%$ & 74 & $100 \%$ & 76 \\
\hline 3,4 & $74 / 76$ & 50 & $67,6 \%$ & 74 & $100 \%$ & 76 \\
\hline 4,5 & $76 / 70$ & 40 & $57,1 \%$ & 70 & $100 \%$ & 76 \\
\hline 5,6 & $70 / 54$ & 32 & $59,3 \%$ & 54 & $100 \%$ & 70 \\
\hline 6,7 & $54 / 46$ & 24 & $52,2 \%$ & 46 & $100 \%$ & 54 \\
\hline 7,8 & $46 / 38$ & 22 & $57,9 \%$ & 38 & $100 \%$ & 46 \\
\hline 8,9 & $38 / 34$ & 17 & $50,0 \%$ & 34 & $100 \%$ & 38 \\
\hline 9,10 & $34 / 34$ & 32 & $94,1 \%$ & 34 & $100 \%$ & \\
\hline 10,11 & $34 / 34$ & 17 & $50,0 \%$ & 34 & $100 \%$ & \\
\hline \hline
\end{tabular}

Table 3: Number ( $\mathrm{N}^{\circ}$ Match) and percentage of matches (\% Match) obtained with the eleven isocontours extracted from the image with ID 6 of the example sequence.

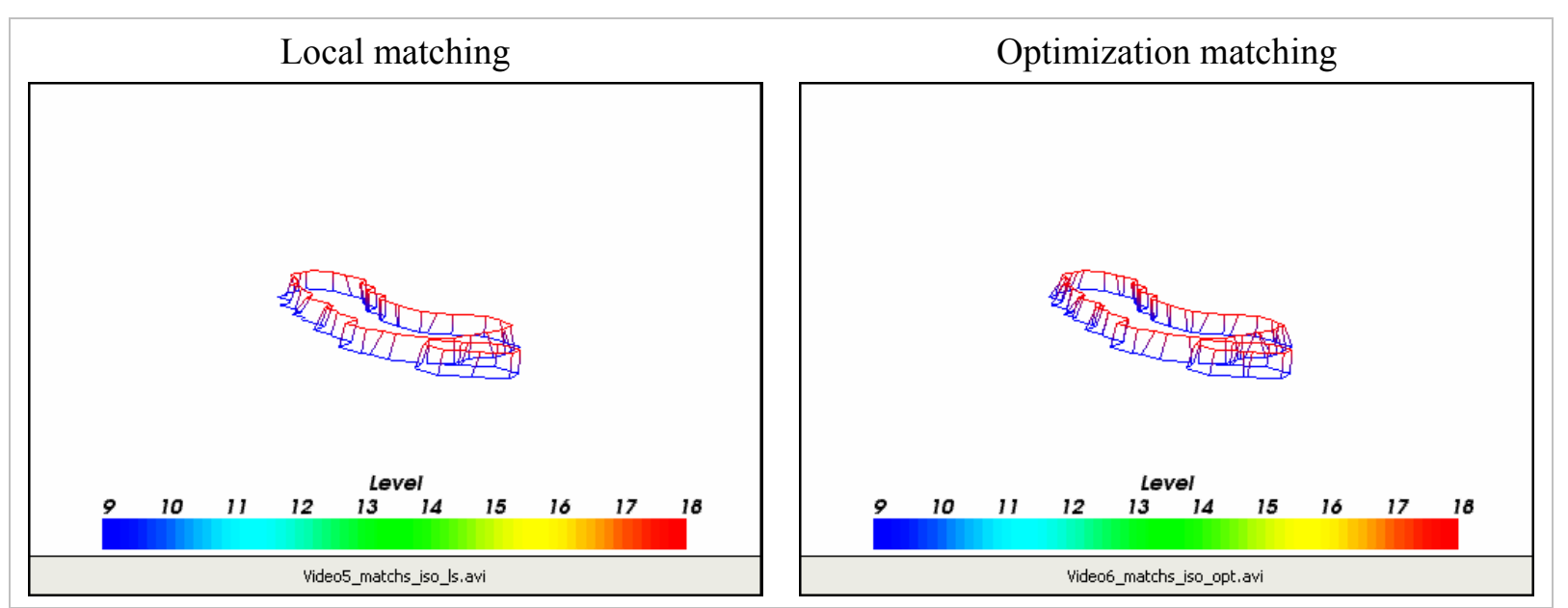

Videos 5 and 6: Matches found between the isocontours extracted from the image with ID 6 of the example sequence (click to see the video).

To have satisfactory matching results in some of the examples considered, when compared with the local search strategy, the global search strategy always required an inferior number of eigenvectors in the affinity matrix construction. This suggests that the total computational effort of the global matching methodology can be reduced if optimization techniques are considered.

In terms of execution time, the optimization algorithm that uses the Hungarian method showed to be of low efficiency. In the several experimental examples performed, the Simplex algorithm for flow problems revealed the most efficient among the optimization algorithms considered. The execution time of $L A P m$ algorithm was higher than the Simplex algorithm for flow problems, even being a more specific algorithm for this type of problems. This can be dew to the interval in which lay the elements of the affinity matrix, [0; 1], since when this algorithm was tested in [27] it revealed the most efficient when the considered interval was $[1 ; 100]$.

In the several experimental tests performed considering contour objects, the implemented algorithm for the determination of correspondences of the excess data points always finds satisfactory matches. That allows us to conclude that the referred algorithm can become an interesting base for the development of new solutions for the determination of matches of type "one to many" and vice versa, and that should be ported to more complex objects (i. e., surfaces).

The experimental results shown in this paper, confirm that the proposed physical methodology can satisfactory match objects represented in dynamic pedobarography images, and that the use of the pixel 
brightness values as a third Cartesian coordinate is very satisfactory, both in terms of its interpretation as pressure, and in solving the problems associated to merging or the splitting of objects.

\section{References}

[1] S. E. Sclaroff and A. Pentland, "Modal Matching for Correspondence and Recognition", IEEE Transactions on Pattern Analysis and Machine Intelligence, vol. 17, pp. 545-561, 1995

[2] J. M. R. S. Tavares, PhD Thesis, "Análise de Movimento de Corpos Deformáveis usando Visão Computacional", in Faculdade de Engenharia da Universidade do Porto, Portugal, 2000

[3] Y. Ohta and T. Kanade, "Stereo by Intra- and Inter-Scanline Search using Dynamic Programming", IEEE Transactions on Pattern Analysis and Machine Intelligence, vol. 7, pp. 139-154, 1985

[4] S. Roy and I. J. Cox, "A Maximum-Flow Formulation of the N-camera Stereo Correspondence Problem", presented at International Conference on Computer Vision (ICCV'98), Bombay, India, 1998

[5] S. Gold, A. Rangarajan, C. P. La, S. Pappu and E. Mjolsness, "New algorithms for 2D and 3D point matching: pose estimation and correspondence", Pattern Recognition, vol. 31, pp. 1019-1031, 1998

[6] C. Silva, PhD Thesis, "3D Motion and Dense Structure Estimation: Representation for Visual Perception and the Interpretation of Occlusions", in Instituto Superior Técnico: Universidade Técnica de Lisboa, 2001

[7] J. L. Maciel and J. P. Costeira, "A Global Solution to Sparse Correspondence Problems", IEEE Transactions on Pattern Analysis and Machine Intelligence, vol. 25, pp. 187-199, 2003

[8] Z. Zhang, "Iterative Point Matching for Registration of Free-Form Curves", INRIA, Technical Report RR-1658, April 1992

[9] M. S. Wu and J. J. Leou, "A Bipartite Matching Approach to Feature Correspondence in Stereo Vision”, Pattern Recognition Letters, vol. 16, pp. 23-31, 1995

[10] J. P. P. Starink and E. Backer, "Finding Point Correspondences using Simulated Annealing", Pattern Recognition, vol. 28, pp. 231-240, 1995

[11] S. Belongie, J. Malik and J. Puzicha, "Shape Matching and Object Recognition using Shape Context", IEEE Transactions on Pattern Analysis and Machine Intelligence, vol. 24, pp. 509-522, 2002

[12] A. J. Padilha, L. A. Serra, S. A. Pires and A. F. N. Silva, "Caracterização Espacio-Temporal de Pressões Plantares em Pedobarografia Dinâmica”, FEUP/INEB, Relatório Interno, 1995

[13] J. M. R. S. Tavares, J. Barbosa and A. Padilha, "Matching Image Objects in Dynamic Pedobarography", presented at 11th Portuguese Conference on Pattern Recognition (RecPad'00), Porto, Portugal, 2000

[14] T. F. Cootes and C. J. Taylor, "Modelling Object Appearance Using The Grey-Level Surface", presented at British Machine Vision Conference (BMVC'94), 1994

[15] B. Moghaddam, C. Nastar and A. P. Pentland, "Bayesian Face Recognition using Deformable Intensity Surfaces", MIT Media Laboratory, Technical Report No 371, 1996

[16] K.-J. Bathe, Finite Element Procedures, Prentice Hall, 1996

[17] S. Graham Kelly, Fundamentals of Mechanical Vibrations, McGraw-Hill, 1993

[18] L. F. Bastos and J. M. R. S. Tavares, "Optimization in Modal Matching for Correspondence of Objects Nodal Points", presented at 7th Portuguese Conference on Biomedical Engineering (BioEng'2003), Fundação Calouste Gulbenkian, Lisboa, Portugal, 2003

[19] F. S. Hillier and G. J. Lieberman, Introduction to Operations Research. Mcgraw-Hill International Editions, 1995

[20] L. F. Bastos, MSc Thesis, "Optimização da Determinação das Correspondências entre Objectos Deformáveis no Espaço Modal", in Faculdades de Engenharia e Ciências: Universidade do Porto, Portugal, 2003

[21] A. Löbel, "MFC - A Network Simplex Implementation", Konrad-Zuse-Zentrum für Informationstechnik Berlin, Division Scientific Computing, Department Optimization, 2000 
[22] A. Volgenant, "Linear and Semi-Assignment Problems: A Core Oriented Approach", Computers and Operations Research, vol. 23, pp. 917-932, 1996

[23] J. M. R. S. Tavares, J. Barbosa and A. Padilha, "Apresentação de um Banco de Desenvolvimento e Ensaio para Objectos Deformáveis", Revista Electrónica de Sistemas de Informação, vol. 1, 2002

[24] R. Davies, Newmat, A matrix library in $C^{++}, 2005$

[25] The VTK User's Guide, Kitware Inc., 2003

[26] W. Schroeder, K. Martin and B. Lorensen, The Visualization Toolkit, 3rd Edition, Kitware Inc, 2002

[27] M. Dell'Amico and P. Tooth, "Algorithms and Codes for Dense Assignment Problems: The State of The Art", Discrete Applied Mathematics, 100, pp. 17-48, 2000 